\title{
Causes and predictors of hospital readmissions in patients older than 65 years hospitalized for heart failure with preserved left ventricular ejection fraction in western Romania
}

\author{
This article was published in the following Dove Press journal: \\ Clinical Interventions in Aging \\ 17 June 2015 \\ Number of times this article has been viewed
}

\author{
Adelina Marioara Mavrea' \\ Tiberiu Dragomir' \\ Diana Aurora Bordejevic' \\ Mirela Cleopatra Tomescu' \\ Oana Ancusa' \\ losif Marincu ${ }^{2}$ \\ 'Cardiology Department, ${ }^{2}$ Department \\ of Epidemiology and Infectious \\ Diseases, "Victor Babes" University \\ of Medicine and Pharmacy, Timisoara, \\ Romania
}

Background: Heart failure with preserved ejection fraction (HFpEF) is more frequent in the elderly and is associated with important economic implications because of repetitive and prolonged hospitalizations, due to both cardiovascular and noncardiovascular causes.

Purpose: To identify the causes, as well as the clinical and biological markers, that could be used as predictors of hospital readmissions in HFpEF patients aged $\geq 65$ years.

Patients and methods: Consecutive eligible patients hospitalized for a first heart failure (HF) episode were prospectively included and divided into one of two age groups (elderly: $\geq 65$ years; and nonelderly: $<65$ years). The clinical features, therapeutic approaches, and clinical outcomes during the 1-year follow-up period were analyzed.

Results: A total of 178 patients were included, with a mean age of $64.6 \pm 8.6$ years; 80 (45\%) were women. A total of 98 patients (55\%) were aged $\geq 65$ years, and 80 (45\%) were aged $<65$ years. In the group aged $\geq 65$ years, 58 patients $(59 \%)$ were women, while in the group aged $<65$ years, 22 patients $(28 \%)$ were women $(P=0.0001)$. During the 1 -year follow-up, no patients died or were lost to follow-up. Moreover, $116(65 \%)$ of the HFpEF patients experienced hospital readmissions. The elderly patients had a significantly higher readmission rate ( $73 \%$ vs $55 \%$, respectively; $P<0.02$ ); readmissions due to aggravated HF were significantly more frequent in this age group ( $41 \%$ vs $18 \%$, respectively; $P<0.002)$. Multivariate logistic regression analysis indicated that the independent predictors of readmission due to $\mathrm{HF}$ aggravation included plasma levels of brain natriuretic peptide $>450 \mathrm{pg} / \mathrm{mL}(P<0.01)$ and N-terminal-pro-brain natriuretic peptide $>477 \mathrm{pg} / \mathrm{mL}(P<0.02)$ in the elderly group, while in the nonelderly group, the independent predictors of this outcome were a New York Heart Association functional class of IV at initial hospitalization $(P<0.04)$, as well as plasma levels of brain natriuretic peptide $>390 \mathrm{pg} / \mathrm{mL}(P=0.03)$ and tumor necrosis factor (TNF)- $\alpha$ $>7.1 \mathrm{pg} / \mathrm{mL}(P<0.001)$. Readmissions due to noncardiovascular causes were independently predicted by plasma levels of TNF- $\alpha>10 \mathrm{pg} / \mathrm{mL}$ in the elderly $(P=0.003)$ and of interleukin (IL) $-6>1.9 \mathrm{pg} / \mathrm{mL}$ in the nonelderly $(P<0.04)$.

Conclusion: We conclude that in HFpEF patients aged $\geq 65$ years, the main cause of rehospitalization during the 1-year follow-up was HF aggravation. The risk of this outcome was independently predicted by increased levels of cardiac peptides, while the risk of noncardiovascular readmissions was predicted by increased levels of inflammatory biomarkers. Increased TNF- $\alpha$ levels predicted both cardiovascular and noncardiovascular readmissions, while increased levels of high-sensitivity $\mathrm{C}$-reactive protein did not predict any of these outcomes in our study.

Keywords: elderly, heart failure with preserved left ventricular ejection fraction, hospital readmissions

\footnotetext{
Correspondence: Mirela Cleopatra

Tomescu; Tiberiu Dragomir Cardiology Department, "Victor Babes" University of Medicine and Pharmacy, Second Eftimie Murgu Square, 30004I-

Timisoara, Romania

Tel +40 7229795 I6; +40 72274 |99|

Fax +40256220636

Email tomescu.mirela@umft.ro; tibidragomir@yahoo.com
} 


\section{Introduction}

Heart failure (HF) is a pathological entity with an increasing incidence in the general population, due to increasing life expectancy. It represents the final stage of cardiovascular diseases and has major social and economic impacts. ${ }^{1}$ The number of hospitalizations for HF is increasing, especially in the elderly. ${ }^{2}$ The emergence of new therapeutic strategies in HF has led to significant improvements in cardiovascular morbidity and mortality in HF patients with reduced left ventricular ejection fraction (HFREF). ${ }^{1}$

Although HF with preserved left ventricular ejection fraction (HFpEF) has an incidence that is comparable to that of HFREF, there were no significant improvements in the prognosis of the latter. This differential response to therapy suggests that HFpEF and HFREF are two distinct entities with fundamentally different pathophysiologies. ${ }^{3} \mathrm{It}$ is known that HFpEF patients are older and present with a greater number of comorbidities. Paulus and Tschope propose a new theoretical framework for understanding HFpEF, which considers that not afterload excess, but inflammation associated with comorbidities (obesity, hypertension, diabetes mellitus [DM], chronic obstructive pulmonary disease [COPD], anemia, and chronic kidney disease [CKD]), plays a central role in the development of ventricular hypertrophy and diastolic dysfunction. ${ }^{4} \mathrm{~A}$ better understanding of the interrelationships between the causes and comorbidities of HFpEF could allow for the development of better therapies, both for the structural aspects of heart disease and for its comorbidities, in order to decrease the burden of frequent hospital readmissions in HFpEF patients. ${ }^{2,5}$

The aim of the present study was to identify the causes and predictors of hospital readmissions in HFpEF patients aged $\geq 65$ years.

\section{Patients and methods}

\section{Patient selection}

The study population consisted of adult patients (aged $\geq 18$ years) admitted to the Cardiology Clinic of Timisoara City Hospital, from January 1, 2013 to December 31, 2013, with a first episode of HFpEF. All eligible patients were prospectively included in the study. The inclusion criteria were as follows: left ventricular ejection fraction (LVEF) $\geq 45 \%$ (twodimensional echocardiography: Simpson's method); E/E' $\geq 15$ (Doppler imaging of the tissues); and brain natriuretic peptide (BNP) levels $>150 \mathrm{pg} / \mathrm{mL}$. ${ }^{6,7}$

The exclusion criteria were as follows: acute coronary syndrome within the last 30 days; acute myocarditis; acute pericarditis; acute pulmonary thromboembolism; pacemaker implant; and the need for cardiovascular surgery.

The patients were divided into one of the two groups according to age at admission (elderly: aged $\geq 65$ years; nonelderly: aged $<65$ years).

\section{Data extraction}

Baseline data were extracted from hospital records and included age, sex, New York Heart Association (NYHA) functional class on admission, the primary cause of HF, laboratory data, 12-leads resting electrocardiogram, echocardiographic data, and medical history. Medical history included data regarding smoking, loneliness, obesity, coronary artery disease (CAD), old myocardial infarction (MI), hypertension, valvular disease, peripheral artery disease, DM, COPD, $\mathrm{CKD}$, a history of stroke, and malignancy. The serum biomarkers determined at inclusion were BNP and N-terminal (NT)-proBNP, which served as markers of left ventricular dysfunction. Tumor necrosis factor (TNF)- $\alpha$, interleukin (IL)-6, and high-sensitivity C-reactive protein (hs-CRP) were markers of systemic inflammation. Other laboratory data collected included: blood cell count; serum hemoglobin; serum glucose; serum creatinine; estimated glomerular filtration rate at admission calculated using the simplified Modification of Diet in Renal Disease formula; ${ }^{7}$ serum electrolytes; and lipidogram. Medical treatment records were completed at discharge and at 1-year follow-up. Prescription of the main therapeutic classes in HF was recorded.

\section{Definition of covariates}

A diagnosis of HFpEF was made based on symptoms, physical signs, chest radiography, echocardiography, and natriuretic peptide values on admission, according to the European Society of Cardiology guidelines for the diagnosis of HF. ${ }^{8}$

Ischemic etiology of HF was considered in patients with a history of CAD, documented MI, or angina. ${ }^{9}$

A patient was considered to have hypertension in the presence of high blood pressure (BP) during hospitalization ( $\geq 140 / 90 \mathrm{mmHg}$ ), a previous diagnosis of hypertension, or normal BP with ongoing antihypertensive therapy..$^{10}$ Valvulopathies were identified by history, physical examination, and echocardiographic data. ${ }^{11}$ Peripheral artery disease diagnosis was based on the patient's history, physical examination, ankle-brachial index, and duplex ultrasound. ${ }^{12} \mathrm{CKD}$ was diagnosed in the presence of a Modification of Diet in Renal Disease estimated glomerular filtration rate $<60 \mathrm{~mL} /$ $\min / 1.73 \mathrm{~m}^{2} .{ }^{8}$ Metabolic syndrome (MS) was defined in 
accordance to the International Diabetes Federation's (IDF) criteria, ie, central obesity (waist circumference $>94 \mathrm{~cm}$ for men; $>80 \mathrm{~cm}$ for women) or a body mass index $>30 \mathrm{~kg} / \mathrm{m}^{2}$, and any two of the following: raised triglycerides, $>150 \mathrm{mg} / \mathrm{dL}$ $(1.7 \mathrm{mmol} / \mathrm{L})$, or specific treatment for this lipid abnormality; reduced high-density lipoprotein cholesterol, $<40 \mathrm{mg} / \mathrm{dL}$ $(1.03 \mathrm{mmol} / \mathrm{L})$ in males, <50 $\mathrm{mg} / \mathrm{dL}(1.29 \mathrm{mmol} / \mathrm{L})$ in females, or specific treatment for this lipid abnormality; raised BP: systolic BP $>130 \mathrm{mmHg}$ and/or diastolic BP $>85 \mathrm{mmHg}$, or the treatment of previously diagnosed hypertension; raised fasting plasma glucose, $>100 \mathrm{mg} / \mathrm{dL}$ (5.6 mmol/L), or previously diagnosed type 2 diabetes. $^{13}$ DM was diagnosed according to World Health Organization (WHO)/IDF, and included the presence of any one of the following: glycated hemoglobin $\left(\mathrm{HbA}_{1 \mathrm{c}}\right) \geq 6.5 \%$; fasting plasma glucose level $\geq 7.0 \mathrm{mmol} / \mathrm{L}(126 \mathrm{mg} / \mathrm{dL})$; or a plasma glucose level $\geq 11 \mathrm{mmol} / \mathrm{L}$ ( $200 \mathrm{mg} / \mathrm{dL}$ ) 2 hours after $75 \mathrm{~g}$ of oral glucose load. ${ }^{14}$ Obesity was defined as a body mass index $\geq 30 \mathrm{~kg} / \mathrm{m}^{2} .{ }^{14}$ We used the WHO's criterion for anemia in adults (an hemoglobin value of $<12.5 \mathrm{~g} / \mathrm{dL}$ ). ${ }^{15}$

COPD diagnosis was based on Global Strategy for Diagnosis, Management, and Prevention of Chronic Obstructive Lung Disease (GOLD) criteria ${ }^{16}$ and they included patients with a history of dyspnea that was persistent and worsening over time, associated with chronic cough or sputum production, and a history of exposure to risk factors for the disease, especially cigarette smoking. The diagnosis of COPD was confirmed by spirometry, showing a persistent airflow limitation.

An echocardiographic evaluation was performed within the first 24 hours of hospitalization using a VIVID S5 echocardiograph. Mono- and two-dimensional imaging, pulsed and continuous Doppler, as well as tissue Doppler imaging were performed in all patients. LVEF was calculated using Simpson's method. After assessing the antegrade mitral flow and tissue Doppler parameters, we determined the $\mathrm{E} / \mathrm{E}^{\prime}$ ratio. ${ }^{17}$

BNP was measured using a fluorescence immunoassay kit (Triage ${ }^{\circledR}$; Biosite Incorporated, San Diego, CA, USA). The measurable range of BNP assays was 5.0-5,000 pg/mL. The day before discharge, patients completed the Minnesota Living with Heart Failure Questionnaire (MLHFQ). ${ }^{18}$ An MLHFQ score $<40$ was interpreted as a slight impairment, a score of 40-70 was considered as moderate, while a score $>70$ was interpreted as a severely impaired quality of life. In order to obtain further information on the patient's mental status, we asked patients to complete the Fahrenberg scale, a questionnaire that assesses the risk of depression. ${ }^{19}$
It contains 14 items, with two response options, "zero" meaning absent and "one" meaning present. There is an increased risk for depression when the global score is $>7$. On the same day that the questionnaires were completed, an exercise test was performed, using the 6-minute walk test (6MWT). ${ }^{20}$ The test was performed in the morning after fasting, with drug therapy administered as planned before this test, and without previous training.

\section{Follow-up and outcomes}

Hospital readmissions and deaths were assessed as adverse outcomes. On discharge, the patients and their families were asked to notify the attending physician as soon as possible in case of health problems requiring emergency medical services or hospitalization. Patients were contacted by telephone 6 months after enrollment to provide information regarding the progress of HF. In the case of hospital admissions, additional information was requested regarding the diagnosis on admission and changes in the treatment plan.

One year after enrollment, the patients were reevaluated by clinical examination and laboratory tests, which included the determination of BNP, NT-proBNP, IL-6, hs-CRP, and TNF- $\alpha$ levels. Patients completed the MLHFQ and Fahrenberg scales. Exercise capacity was reassessed by $6 \mathrm{MWT}$. At the same time, the patients were requested to provide their medical information; moreover, documentation on their health evolution, from the time of enrollment in the study until the end of the follow-up period, was completed.

All causes of readmission were registered during the follow-up period of 1 year. The causes of readmission were assessed by the documentation of hospital records. Cardiovascular readmissions were divided into readmissions due to worsening HF and other cardiovascular causes. Worsening HF readmissions were considered as those who had an admission diagnosis of acute pulmonary edema, cardiogenic shock, decompensated cardiac failure, or those who required intravenous administration of furosemide $\geq 2$ vials at admission. The symptoms of worsening HF had to include at least one of the following: progressive exercise dyspnea and/or fatigue; orthopnea; or paroxysmal nocturnal dyspnea. In addition, at least two of the following signs also had to be present: leg edema; rapid increase in weight by fluid retention; jugular veins distension; hepatomegaly; pulmonary rales; pulmonary stasis on chest radiography; a third heart sound; or an increase in BNP or NT-proBNP level. Readmissions due to other cardiovascular causes included hospitalizations due to atrial fibrillation, hypertensive crisis, acute coronary syndrome, pulmonary embolism, 
stroke, or acute peripheral ischemia. Other causes of readmissions were classified as noncardiovascular readmissions. No patients died during the study.

\section{Statistical analysis}

Data were collected and analyzed using the MedCalc 12.3.0.0 statistical software for Windows. Data are given as the mean \pm 1 standard deviation when normally distributed, as the median and interquartile range when the distribution was not normal or skewed, and as frequencies and percentages for categorical variables. The associations between baseline variables were evaluated by means of one-way analysis of variance, the Mann-Whitney test, and the chi-square or Fisher's exact test, as appropriate. The association of variables with follow-up outcomes was analyzed by calculating the odds ratio (OR) together with the confidence intervals in univariate logistic regression analysis. Multivariate analysis included the significant independent variables at univariate analysis. For the independent predictors identified by multivariate stepwise logistical regression, a receiver operating characteristic (ROC) analysis was performed. The covariates that were considered to have a potential prognostic impact included age; sex; loneliness; NYHA functional class; ischemic etiology of HF; a history of hypertension, CAD, old MI, atrial fibrillation, heart rate at admission, peripheral artery disease, DM, obesity, MS, CKD, COPD, cancer, stroke, degenerative osteoarthritis, or anemia; quality of life with HF assessed by the MLHFQ; a risk of depression evaluated by Fahrenberg scale; the values of natriuretic peptides BNP and NT-proBNP; as well as the levels of inflammatory markers hs-CRP, IL-6, and TNF- $\alpha$. A $P$-value $<0.05$ was considered as the threshold for statistical significance. All $P$-values are the results of two-tailed tests.

\section{Ethics}

The study was advised by the ethics commission at our hospital. Before enrollment, all patients provided their signed informed consent for participation in the study, according to the Human Rights Declaration of Helsinki. Before obtaining consent, the patient was given sufficient time and opportunity to inquire about the details of the study and he or she decided whether or not to participate in the study.

\section{Results}

The study population included 178 patients, $45 \%$ of which were women. The mean age was $64 \pm 8$ years. Of the 178 patients, $98(55 \%)$ were aged $\geq 65$ years; of those in this age group, 58 (59\%) were women; conversely, 80 (45\%) were aged $<65$ years, and $22(28 \%)$ of these patients were women. All patients were hospitalized with a first diagnosis of HF. The average length of stay was $7 \pm 3$ days. The demographic, clinical, and biological data of the patients are listed in Table 1. The comorbidities included: hypertension in $136(76 \%)$, CAD in 108 (61\%), DM in $57(32 \%)$, MS in 102 (57\%), CKD in 87 (49\%), COPD in 44 (25\%), obesity in $85(48 \%)$, peripheral artery disease in $26(15 \%)$, stroke in eleven (6\%), anemia in $30(17 \%)$, degenerative osteoarthritis in $56(31 \%)$, and cancer history in four $(2 \%)$ patients. The treatment of patients consisted of beta blockers in $152(85 \%)$, angiotensin-converting enzyme (ACE) inhibitors in 101 (55\%), angiotensin receptor blockers (ARBs) in 51 (29\%), ivabradine in $70(39 \%)$, diuretics in 129 (72\%), and statins in $114(64 \%)$ patients. The elderly patients who were admitted with HFpEF were, when compared to those aged $<65$ years, significantly more likely to be women $(P=0.0001)$, and they were also more likely to have a NYHA functional class of IV $(P<0.001)$. These patients had a higher prevalence of hypertension $(P<0.0001)$, obesity $(P=0.0002)$, MS $(P<0.0001)$, and $\mathrm{CKD}(P<0.0001)$. The elderly patients had lower heart rate values at admission $(P=0.001)$, and they were more likely to present with atrial fibrillation $(P=0.0001)$. They most often received treatment with diuretics $(P<0.0001)$. The values of the inflammatory markers were significantly higher in the elderly $(P<0.0001$ for TNF- $\alpha$ and hs-CRP; $P=0.0001$ for IL-6), while the values of the natriuretic peptides were not significantly different when compared to that in the nonelderly HFpEF patients. The elderly patients had, at baseline, a significantly poorer quality of life $(P=0.005)$, a higher risk for depression $(P=0.002)$, and a poorer 6MWT score $(P=0.029)$ (Table 1$)$.

None of the patients enrolled in the study died during the initial hospitalization or during the follow-up period. No patients were lost to follow-up. At the 1-year evaluation, the values of the cardiac peptides BNP and NT-proBNP did not differ significantly between the two age groups of HF patients. The values of the inflammatory biomarkers were significantly higher in the elderly group $(P<0.0001$ for TNF- $\alpha$ and hs-CRP; $P=0.0001$ for IL-6). Quality of life, as assessed by the MLHFQ, was similar, although the elderly patients had a significantly shorter distance walked on the 6MWT $(P<0.03)$ and a significantly higher risk for depression $(P<0.003)$, as shown in Table 2.

During the 1-year follow-up, $116(65 \%)$ patients were hospitalized. Of the readmissions, 54 (30\%) were caused by HF aggravation, 35 (20\%) had other cardiovascular causes, and $27(15 \%)$ had noncardiovascular causes. In the elderly 
Table I Differences between HFpEF patients according to age, at baseline

\begin{tabular}{|c|c|c|c|}
\hline Characteristics & Age $\geq 65$ years $(n=98)$ & Age $<65$ years $(n=80)$ & $P$-value \\
\hline Women & $58(59)$ & $22(28)$ & $0.0001 *$ \\
\hline Lonely & $45(46)$ & $30(38)$ & 0.3566 \\
\hline \multicolumn{4}{|l|}{ Primary etiology of $\mathrm{HF}$} \\
\hline Ischemic & $20(22)$ & $27(34)$ & 0.1059 \\
\hline Non-ischemic & $76(78)$ & $53(66)$ & 0.1059 \\
\hline \multicolumn{4}{|l|}{ NYHA class } \\
\hline III & $20(21)$ & $47(46)$ & $0.0007^{*}$ \\
\hline IV & $78(79)$ & $33(54)$ & $0.0007^{*}$ \\
\hline Hypertension & $86(88)$ & $50(63)$ & $<0.000 I^{*}$ \\
\hline CAD & $54(55)$ & $54(80)$ & $0.0005^{*}$ \\
\hline Old MI & $23(24)$ & $16(20)$ & 0.6473 \\
\hline Atrial fibrillation & $50(52)$ & $20(26)$ & $0.0001 *$ \\
\hline Heart rate at admission (bpm) & $75 \pm 16$ & $83 \pm 17$ & $0.0015^{*}$ \\
\hline DM & $33(34)$ & $24(30)$ & 0.2498 \\
\hline Metabolic syndrome & $70(72)$ & $32(40)$ & $<0.000 I^{*}$ \\
\hline Obesity & $60(61)$ & $25(32)$ & $0.0002 *$ \\
\hline CKD & $62(63)$ & $25(3 \mathrm{I})$ & $<0.000 I^{*}$ \\
\hline COPD & $27(28)$ & $17(2 \mid)$ & 0.3678 \\
\hline Peripheral artery disease & $17(16)$ & $9(12)$ & 0.5860 \\
\hline Anemia & $19(20)$ & $11(14)$ & 0.3934 \\
\hline Degenerative osteoarthritis & $34(35)$ & $22(28)$ & 0.4035 \\
\hline History of stroke & $7(7)$ & $4(5)$ & 0.8105 \\
\hline History of cancer & $3(3)$ & $\mathrm{I}(\mathrm{I})$ & 0.689 \\
\hline ACE-I & $48(49)$ & $49(62)$ & 0.1136 \\
\hline ARBs & $34(35)$ & $17(2 \mid)$ & 0.0591 \\
\hline Beta blockers & $86(88)$ & $66(83)$ & 0.4631 \\
\hline Ivabradine & $37(38)$ & $33(42)$ & 0.6977 \\
\hline Furosemide & $84(86)$ & $45(57)$ & $<0.000 I^{*}$ \\
\hline Spironolactone & $84(86)$ & $45(57)$ & $<0.000 I^{*}$ \\
\hline Statins & $52(53)$ & $62(78)$ & $0.0010^{*}$ \\
\hline $\mathrm{BNP}(\mathrm{pg} / \mathrm{mL})$ & $428(3 \mid 2-522)$ & $390(30 \mathrm{I}-547)$ & 0.6672 \\
\hline NT-proBNP (pg/mL) & $730(376-973)$ & $485(322-885)$ & 0.2324 \\
\hline TNF- $\alpha(p g / m L)$ & $10.3(9.0-13.0)$ & $7.2(5.8-12.0)$ & $<0.000 I^{*}$ \\
\hline IL-6 (pg/mL) & $4.5(1.8-5.4)$ & $2.2(1.5-3.0)$ & $0.0001 *$ \\
\hline hs-CRP (mg/L) & $5.8(3.2-18.5)$ & $2.5(1.2-2.9)$ & $<0.000 I^{*}$ \\
\hline MLHFQ score & $48(44-53)$ & $42(33-64)$ & $0.0052 *$ \\
\hline 6MWT (m) & $255(165-282)$ & $259(220-319)$ & $0.0293 *$ \\
\hline Fahrenberg scale score & $8.0(7.0-10.0)$ & $7.0(6.0-9.0)$ & $0.0024 *$ \\
\hline
\end{tabular}

Notes: Categorical variables are presented as number (\%). Continuous data are presented as mean \pm I standard deviation when normally distributed and as median (interquartile range) when skewed. ${ }^{*} P$-value $<0.05$.

Abbreviations: HFpEF, heart failure with preserved ejection fraction; HF, heart failure; NYHA, New York Heart Association; CAD, coronary artery disease; bpm, beats per minute; MI, myocardial infarction; DM, diabetes mellitus; CKD, chronic kidney disease; COPD, chronic obstructive pulmonary disease; ACE-I, angiotensin-converting enzyme inhibitors; ARBs, angiotensin receptor blockers; BNP, brain natriuretic peptide; NT-proBNP, N-terminal-pro-brain natriuretic peptide; TNF, tumor necrosis factor;

IL, interleukin; hs-CRP, high-sensitivity C-reactive protein; MLHFQ, Minnesota living with heart failure questionnaire; 6MWT, 6-minute walk test.

group, 72 patients $(73 \%)$ were hospitalized: $40(41 \%)$ due to HF aggravation; $12(12 \%)$ due to other cardiovascular causes; and 20 (20\%) due to noncardiovascular causes. In the nonelderly group, 44 (55\%) of the patients were hospitalized during the 1-year follow-up: 14 (18\%) due to HF aggravation; $23(28 \%)$ due to other cardiovascular causes; and seven (9\%) due to noncardiovascular causes (Table 2).

The total readmission rate was significantly higher in the elderly group $(P<0.02)$. Readmissions due to aggravated HF were significantly more frequent among the elderly patients
(41\% vs $18 \%$, respectively; $P<0.002$ ), while readmissions due to other cardiovascular causes were more frequent in the nonelderly patients ( $28 \%$ vs $12 \%$, respectively; $P<0.02$ ). Readmissions due to noncardiovascular causes were slightly higher in the elderly group ( $20 \%$ vs $9 \%$, respectively; $P=0.06$ ), as shown in Table 2 .

Using the multivariate stepwise logistic regression analysis (Table 3), we found that the variables independently associated with readmissions during the 1-year follow-up in the elderly HFpEF patients were: a BNP level $>450 \mathrm{pg} / \mathrm{mL}$ (sensitivity 
Table 2 Differences between HFpEF patients according to age, at I-year follow-up

\begin{tabular}{|c|c|c|c|}
\hline Characteristics & Age $\geq 65$ years $(n=98)$ & Age $<65$ years $(n=80)$ & $P$-value \\
\hline Total readmissions & $72(73)$ & $44(55)$ & $0.0190 *$ \\
\hline Heart failure aggravation & $40(4 I)$ & $14(18)$ & $0.0016 *$ \\
\hline Other cardiovascular reasons & $12(12)$ & $23(28)$ & $0.0122 *$ \\
\hline Noncardiovascular readmissions & $20(20)$ & $7(9)$ & 0.0671 \\
\hline $\mathrm{BNP}(\mathrm{pg} / \mathrm{mL})$ & $345(214-598)$ & $245(267-435)$ & 0.425 \\
\hline NT-proBNP (pg/mL) & 753 (379-929) & 485 (322-973) & 0.406 \\
\hline TNF- $\alpha(\mathrm{pg} / \mathrm{mL})$ & $9.1(7.6-13.0)$ & $6.2(4.8-11.0)$ & $<0.0001 *$ \\
\hline IL-6 (pg/mL) & $4.0(1.9-5.4)$ & $2.2(1.5-3.0)$ & $0.0001 *$ \\
\hline $\mathrm{hs}-\mathrm{CRP}(\mathrm{mg} / \mathrm{L})$ & $6.6(3.7-22.2)$ & $2.5(1.0-2.9)$ & $<0.000 I^{*}$ \\
\hline MLHFQ score & $46(39-7 I)$ & $42(33-64.5)$ & 0.0697 \\
\hline 6MWT (m) & $255(185-282)$ & $259(220-319)$ & $0.0293 *$ \\
\hline Fahrenberg scale score & $8.0(7.0-10.0)$ & $7.0(6.0-9.0)$ & $0.0024 *$ \\
\hline
\end{tabular}

Notes: Categorical variables are presented as number (\%). Continuous data are presented as mean $\pm \mathrm{I}$ standard deviation when normally distributed and as median (interquartile range) when skewed. ${ }^{*} P$-value $<0.05$.

Abbreviations: HFpEF, heart failure with preserved ejection fraction; BNP, brain natriuretic peptide; NT-proBNP, N-terminal-pro-brain natriuretic peptide; TNF, tumor necrosis factor; IL, interleukin; hs-CRP, high-sensitivity C-reactive protein; MLHFQ, Minnesota living with heart failure questionnaire; 6MWT, 6-minute walk test.

Table 3 Predictors for readmissions in HFpEF patients

\begin{tabular}{|c|c|c|c|c|}
\hline Readmissions & Odds ratio & Standard error & $P$-value & $95 \% \mathrm{Cl}$ \\
\hline \multicolumn{5}{|c|}{ Readmissions in HFpEF aged $\geq 65$ years } \\
\hline BNP $>450$ pg/mL (yes vs no) & 0.891 & 0.047 & $<0.0001$ & $0.767-0.963$ \\
\hline Fahrenberg score $>7.4$ (yes vs no) & 0.713 & 0.074 & 0.002 & $0.565-0.835$ \\
\hline $6 \mathrm{MWT} \leq 248 \mathrm{~m}$ (yes vs no) & 0.712 & 0.087 & 0.009 & $0.564-0.834$ \\
\hline TNF- $\alpha \geq 10.1 \mathrm{pg} / \mathrm{mL}$ (yes vs no) & 0.648 & 0.085 & 0.027 & $0.497-0.780$ \\
\hline \multicolumn{5}{|c|}{ Readmissions in HFpEF aged $<65$ years } \\
\hline NYHA class IV (yes vs no) & 0.038 & 1.486 & 0.027 & $0.002-0.698$ \\
\hline CAD (yes vs no) & 2.931 & 0.517 & 0.037 & $1.063-8.079$ \\
\hline DM (yes vs no) & 5.044 & 0.536 & 0.002 & $1.764-14.423$ \\
\hline CKD (yes vs no) & 0.703 & 0.086 & 0.034 & $0.502-0.806$ \\
\hline Fahrenberg score $>7.4$ (yes vs no) & 0.584 & 0.229 & 0.005 & $0.339-0.832$ \\
\hline $\mathrm{IL}-6 \geq 2.5 \mathrm{pg} / \mathrm{mL}$ (yes vs no) & 2.069 & 0.250 & 0.003 & $1.266-3.38$ \\
\hline TNF- $\alpha>7.1 \mathrm{pg} / \mathrm{mL}$ (yes vs no) & 1.584 & 0.138 & $<0.001$ & $1.207-2.078$ \\
\hline \multicolumn{5}{|c|}{ Readmissions due to cardiovascular causes in the elderly HFpEF patients } \\
\hline NT-proBNP >458 pg/mL (yes vs no) & 1.002 & 0.001 & 0.001 & $1.001-1.004$ \\
\hline Fahrenberg score $>7.4$ (yes vs no) & 1.444 & 1.346 & 0.006 & $1.109-1.880$ \\
\hline \multicolumn{5}{|c|}{ Readmissions due to HF aggravation in the elderly HFpEF patients } \\
\hline BNP $>450 \mathrm{pg} / \mathrm{mL}$ (yes vs no) & 1.004 & 0.001 & 0.007 & $1.001-1.007$ \\
\hline NT-proBNP $>477$ pg/mL (yes vs no) & 1.002 & 0.008 & 0.013 & $1.004-1.003$ \\
\hline \multicolumn{5}{|c|}{ Readmissions due to HF aggravation in the nonelderly HFpEF patients } \\
\hline BNP $>390 \mathrm{pg} / \mathrm{mL}$ (yes vs no) & 1.002 & 0.001 & 0.030 & $1.003-1.005$ \\
\hline TNF- $\alpha>7.1 \mathrm{pg} / \mathrm{mL}$ (yes vs no) & $1.37 \mid$ & 0.082 & $<0.001$ & $1.167-1.610$ \\
\hline \multicolumn{5}{|c|}{ Readmissions due to other cardiovascular causes in nonelderly HFpEF patients } \\
\hline MLHFQ score $>46$ (yes vs no) & 2.705 & 0.448 & $0.04 I$ & I.038-7.050 \\
\hline IL-6 $>2.1 \mathrm{pg} / \mathrm{mL}$ (yes vs no) & 0.537 & 0.313 & 0.047 & $0.290-0.993$ \\
\hline \multicolumn{5}{|c|}{ Readmissions due to noncardiovascular causes in the elderly } \\
\hline TNF- $\alpha>10$ pg/mL (yes vs no) & 1.300 & 0.888 & 0.003 & $1.092-1.547$ \\
\hline \multicolumn{5}{|c|}{ Readmissions due to noncardiovascular causes in the nonelderly } \\
\hline $\mathrm{IL}-6>1.9 \mathrm{pg} / \mathrm{mL}$ (yes vs no) & 0.274 & 0.627 & 0.039 & $0.080-0.940$ \\
\hline
\end{tabular}


of $73 \%$; specificity of $66 \%$ ); a TNF- $\alpha$ level $\geq 10.1 \mathrm{pg} / \mathrm{mL}$ (sensitivity of $64 \%$; specificity of $52 \%$ ); a risk for depression score $>7.4$ (sensitivity of $62 \%$; specificity of $59 \%$ ); and a distance on the 6MWT of $\leq 248 \mathrm{~m}$ (sensitivity of $68 \%$; specificity of $64 \%$ ). The ROC curves of these parameters are presented in Figure 1.

In the nonelderly HFpEF patients, readmissions during the 1-year follow-up period were independently predicted by the following variables: a NYHA functional class of IV at baseline hospitalization; the presence of CAD, DM, or CKD; a risk for depression score $\geq 7.4$ (sensitivity of $76 \%$; specificity of $50 \%$ ); an IL-6 level $\geq 2.5 \mathrm{pg} / \mathrm{mL}$ (sensitivity of $67 \%$; specificity of $30 \%$ ); and a TNF- $\alpha$ level $>7.1 \mathrm{pg} / \mathrm{mL}$ (sensitivity of $58 \%$; specificity of $61 \%$ ), (area under the curve $=0.670$ ). The ROC curves of these parameters are illustrated in Figure 2.

Readmissions due to cardiovascular causes in the elderly were independently predicted by NT-proBNP levels $>458 \mathrm{pg} / \mathrm{mL}$ (sensitivity of $74 \%$; specificity of $71 \%$ ) and by a risk for depression score $\geq 7.4$ (sensitivity of $47 \%$; specificity of 58\%) (Figure 3), while in the nonelderly no independent predictors were retained in the model.

The independent variables predicting readmissions due to HF aggravation in the elderly were a BNP level $>450 \mathrm{pg} / \mathrm{mL}$ (sensitivity of $73 \%$; specificity of $66 \%$ ) and an NT-proBNP level $>477 \mathrm{pg} / \mathrm{mL}$ (sensitivity of $68 \%$; specificity of $60 \%$ ). The ROC curves of these variables are shown in Figure 4 . In the

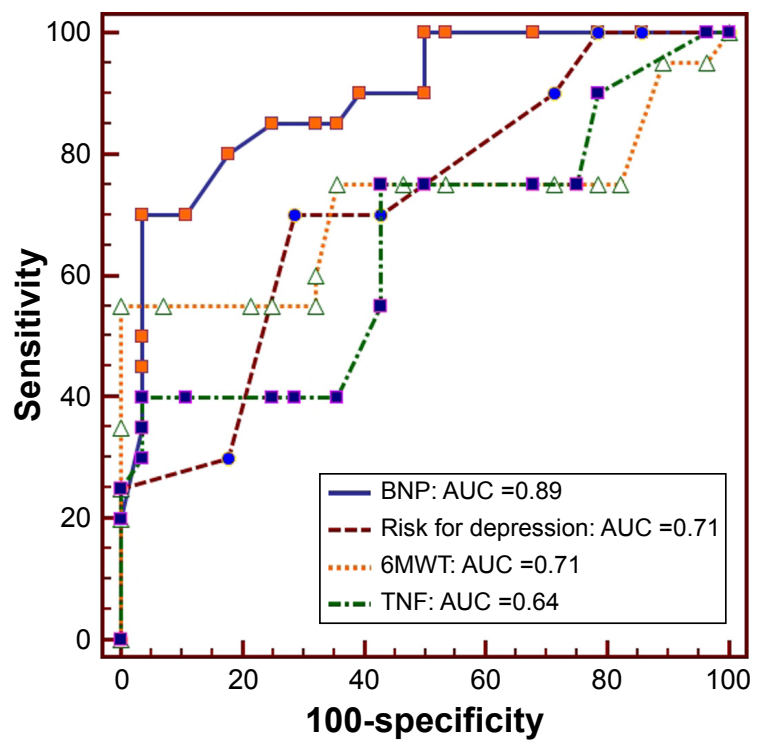

Figure I Receiver operating characteristic (ROC) curves for hospital readmission predictors in HFpEF aged $\geq 65$ years.

Abbreviations: $\mathrm{HFpEF}$, heart failure with preserved ejection fraction; BNP, brain natriuretic peptide; 6MWT, 6-minute walk test; TNF, tumor necrosis factor; AUC, area under the curve.

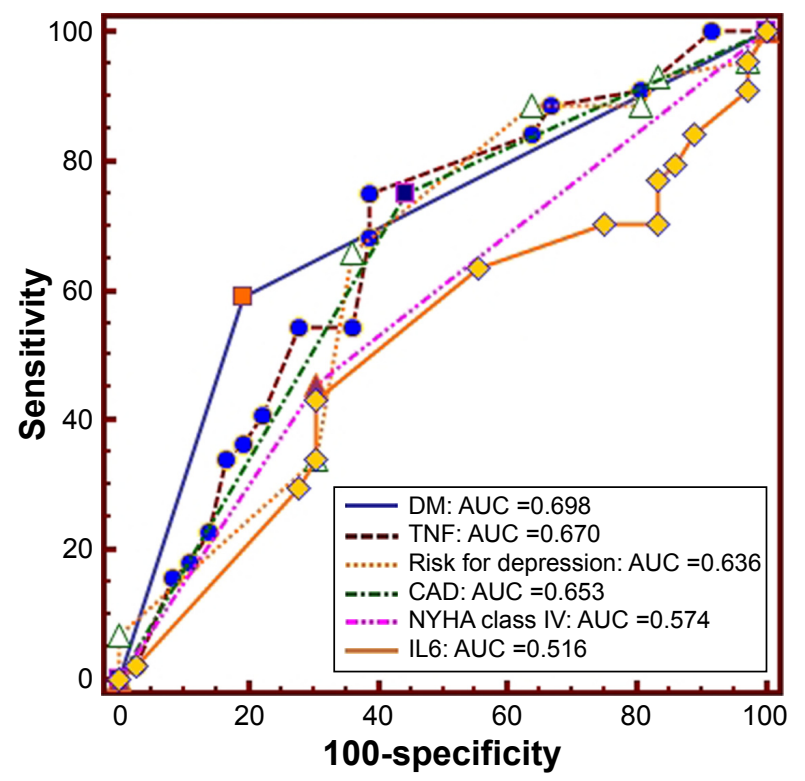

Figure 2 Receiver operating characteristic (ROC) curves for hospital readmission predictors in HFpEF patients aged $<65$ years.

Abbreviations: $\mathrm{HFpEF}$, heart failure with preserved ejection fraction; DM, diabetes mellitus; TNF, tumor necrosis factor; CAD, coronary artery disease; NYHA, New York Heart Association; IL, interleukin; AUC, area under the curve.

nonelderly HFpEF patients, HF aggravation readmissions were independently predicted by BNP levels $>390 \mathrm{pg} / \mathrm{mL}$ (sensitivity of $61 \%$; specificity of $56 \%$ ) and by a TNF- $\alpha$ level $>7.1 \mathrm{pg} / \mathrm{mL}$ (sensitivity of $85 \%$; specificity of $59 \%$ ), as shown in Figure 5.

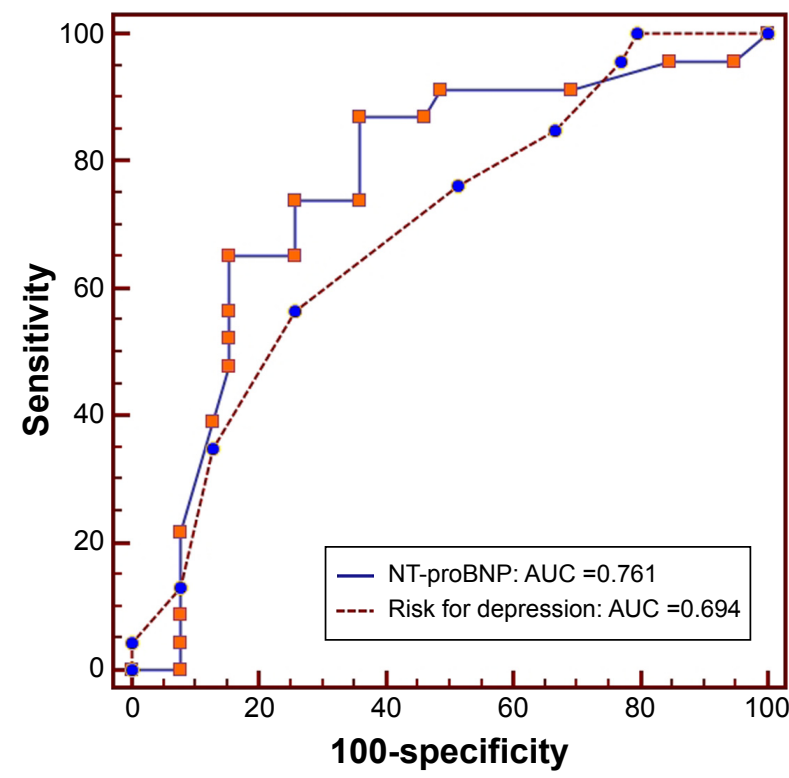

Figure 3 Receiver operating characteristic (ROC) curves for readmissions due to cardiovascular causes in HFpEF patients aged $\geq 65$ years.

Abbreviations: $\mathrm{HFpEF}$, heart failure with preserved ejection fraction; NT-proBNP, $\mathrm{N}$-terminal-pro-brain natriuretic peptide; AUC, area under the curve. 


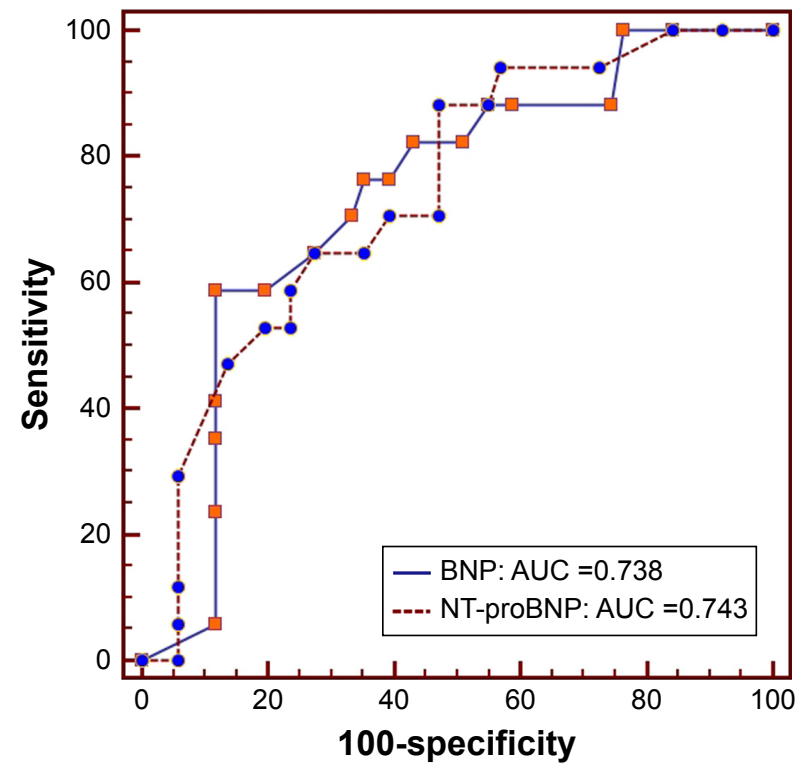

Figure 4 Receiver operating characteristic (ROC) curves for readmissions due to heart failure aggravation in $\mathrm{HFPEF}$ patients aged $\geq 65$ years.

Abbreviations: $\mathrm{HFpEF}$, heart failure with preserved ejection fraction; BNP, brain natriuretic peptide; NT-proBNP, N-terminal-pro-brain natriuretic peptide; AUC, area under the curve.

No variables were found to predict other cardiovascular readmissions in the elderly. In the nonelderly, the model retained the following variables as independent predictors: an MLHFQ score $>46$ (sensitivity of $74 \%$; specificity of $70 \%$ ); and IL-6 level $\geq 2,1 \mathrm{pg} / \mathrm{mL}$ (sensitivity of $63 \%$; specificity of $45 \%$ ), as shown in Figure 6.

Noncardiovascular readmissions in the elderly HFpEF patients were independently predicted by the inflammatory

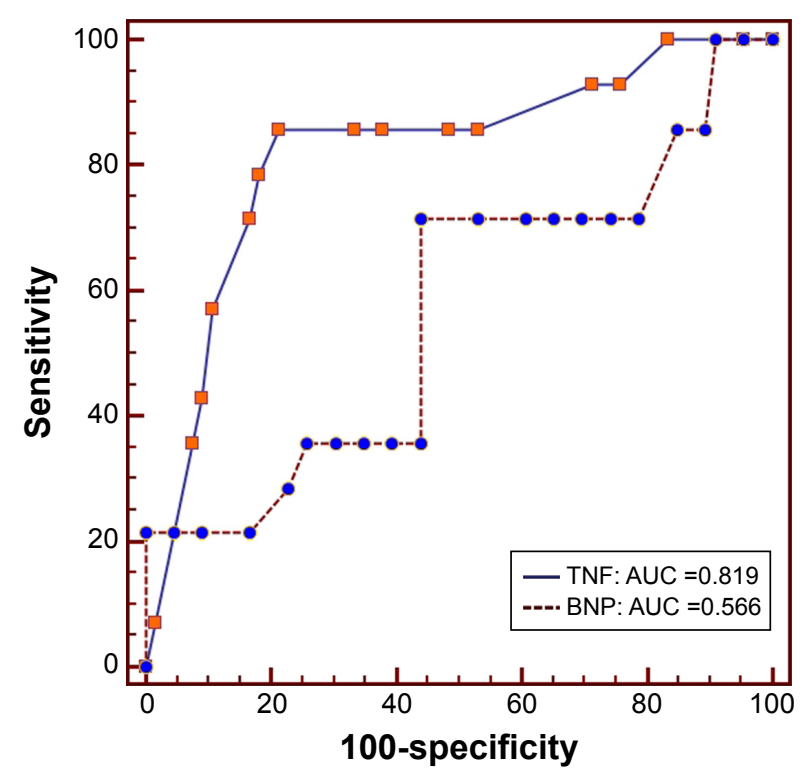

Figure 5 Receiver operating characteristic (ROC) curves for readmissions due to heart failure aggravation in HFpEF patients aged $<65$ years.

Abbreviations: $\mathrm{HFpEF}$, heart failure with preserved ejection fraction; TNF, tumor necrosis factor; BNP, brain natriuretic peptide; AUC, area under the curve.

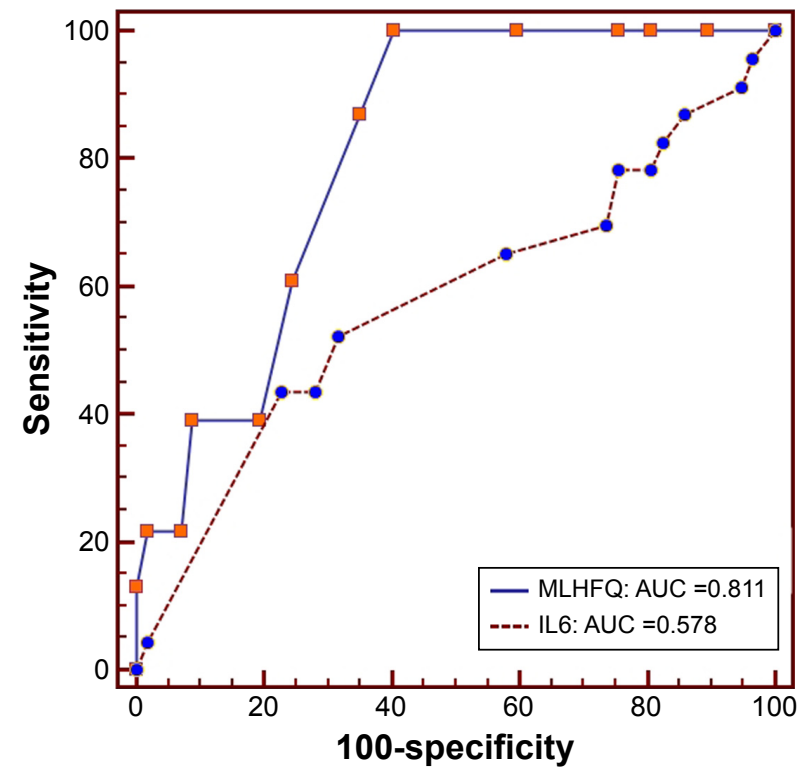

Figure 6 Receiver operating characteristic (ROC) curves for readmissions due to other cardiovascular causes in HFpEF patients aged $<65$ years.

Abbreviations: HFpEF, heart failure with preserved ejection fraction; MLHFQ, Minnesota living with heart failure questionnaire; IL, interleukin; AUC, area under the curve.

marker levels (TNF- $\alpha>10 \mathrm{pg} / \mathrm{mL}$ ) (sensitivity of $67 \%$; specificity of $58 \%$ ). The area under the curve of the ROC curve was 0.691 (Figure 7A). In the nonelderly group, the noncardiovascular readmissions were independently predicted by an IL-6 level $>1.9 \mathrm{pg} / \mathrm{mL}$ (sensitivity of $65 \%$; specificity of $70 \%$ ); the ROC curve is presented in Figure 7B.

\section{Discussion}

Although HF is primarily a disorder of the elderly, there is a lack of evidence-based data from randomized clinical trials of HF. In most clinical trials, the elderly are under-represented, their proportion being between $30 \%$ and $50 \% .^{21,22}$ In addition, the studies that include the elderly are carried out almost exclusively in patients with HF and among those presenting with reduced LVEF. It is possible that the results of these clinical trials do not reflect the real prognosis of elderly HFpEF patients.

Our study was exclusively hospital based, with prospective recruitment of all patients hospitalized for a first episode of HFpEF. Echocardiography was performed in all patients during the first 24 hours after hospital admission.

The included HFpEF patients had a mean age of $64 \pm 8$ years. The mean age was less than that in other studies that have included HFpEF patients (67 years in DIG-PEF; 76 years in SENIORS) ${ }^{22,23}$ A higher mean age was reported in some HF registries; one of the most recent investigations has reported a mean age of 78 years. ${ }^{24}$ We chose to use a cutoff age of 
A

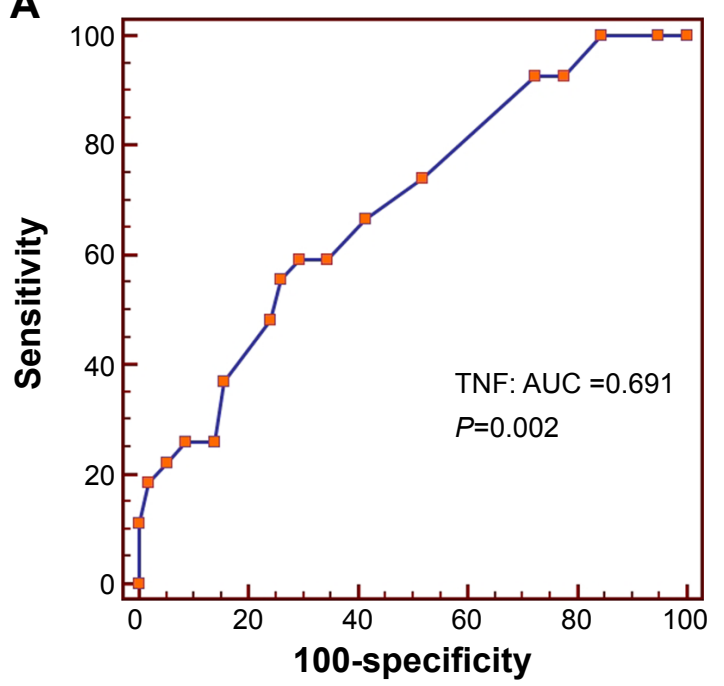

B

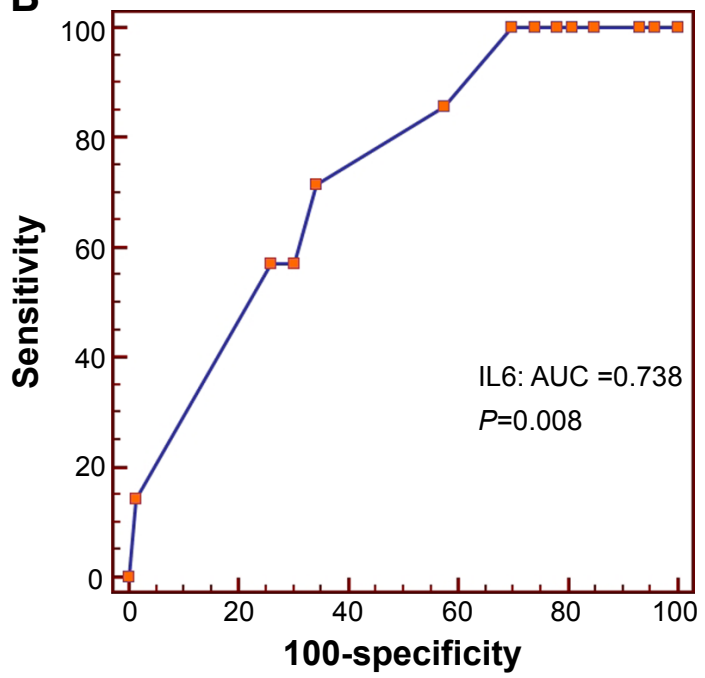

Figure 7 Receiver operating characteristic (ROC) curves for readmissions due to noncardiovascular causes in HFpEF patients aged $\geq 65$ years (A) and $<65$ years (B). Abbreviations: HFpEF, heart failure with preserved ejection fraction; TNF, tumor necrosis factor; IL, interleukin; AUC, area under the curve.

65 years as our definition of the elderly, given that in developed countries, this age coincides with retirement; this age is also considered by the WHO as the onset of old age. ${ }^{25}$

Similar to other HFpEF trials, ${ }^{26,27}$ the elderly patients included in our study were characterized - when compared to HFpEF patients aged $<65$ years - by the following: a higher prevalence of females $(P=0.0001)$; the presence of comorbidities (obesity, $P<0.001$; hypertension, $P<0.0001$; CKD,$P<0.0001)$; and a NYHA functional class of IV $(P<0.001)$. The elderly patients in our study had a poorer physical condition $(P=0.029)$, and the questionnaires that were administered indicated that these patients experienced a poorer quality of life $(P=0.005)$ and were at a higher risk for depression $(P=0.002)$.

The therapeutic attitudes among the elderly patients were different when compared to those of the nonelderly HFpEF patients, but only regarding the higher prescription rate of diuretics ( $86 \%$ vs $57 \%$, respectively; $P<0.0001$ ). This fact can be explained by the higher prevalence of a NYHA class of IV in this group. The prescription rate of beta blockers in our study was $88 \%$ in the elderly and $83 \%$ in the nonelderly, which is higher than that reported in the CHARM-Preserved trial (55\%), I-PRESERVE study (59\%), or the EuroHeart Failure Survey $(39 \%) .{ }^{28,29}$ Beta blockers are known to reduce heart rate, thus leading to improved filling of the left ventricle, which may counterbalance the increased resistance to diastolic filling due to increased stiffness. ${ }^{30}$ Beta blocker therapy was associated with reduced HF mortality in HFREF, but not in HFpEF patients. ${ }^{31}$ Ivabradine was used in $38 \%$ of the elderly and in $42 \%$ of the nonelderly patients $(P=0.697)$ in order to reduce the heart rate. Reil et al demonstrated, in a mouse model of HF with preserved ejection fraction, that heart rate reduction by ivabradine improved diastolic function. ${ }^{32}$ A clinical randomized, double-blind, placebo-controlled study was initiated in 2013 to evaluate the effects of ivabradine on cardiac function, exercise capacity, and neuroendocrine activation in chronic HFpEF. ${ }^{33}$ The prescription rate of ACE inhibitors was $49 \%$ in the elderly and $66 \%$ in the nonelderly, while ARBs were used in 35\% of the elderly and in $21 \%$ of the nonelderly; these rates are comparable to the rates in other clinical trials and registries of HF. ${ }^{28,29}$ Both ACE inhibitors and ARBs have been shown to improve cardiac relaxation and distensibility, and to induce regression of left ventricular hypertrophy; ${ }^{34}$ however, large trials failed to show a clear benefit of these therapeutic agents in HFpEF. ${ }^{35}$

Concerning the biomarkers, the elderly HFpEF patients in our study had higher values of BNPs, both at baseline and at the 1-year follow-up. The natriuretic peptides, which reflect an afterload excess, were independently associated with rehospitalizations due to cardiovascular causes, especially due to HF aggravation $(P=0.001$ for a $\mathrm{BNP}$ level $>450 \mathrm{pg} / \mathrm{mL}$, and $P=0.008$ for an NT-proBNP level $>477 \mathrm{pg} / \mathrm{mL}$ ). Overall, patients with HFpEF have lower levels of BNP and NT-proBNP than do HF patients with reduced LVEF, but for a given BNP level, the associated risk of all-cause mortality and HF hospitalization was shown to be at least as high as in patients with low LVEF. In a study published by van Veldhuisen et al the HFpEF patients in the middle BNP value group (251-750 pg/mL; median: $450 \mathrm{pg} / \mathrm{mL}$ ) had a hazard ratio of 1.50 for death and HF hospitalization. ${ }^{36}$ The findings that BNP is the primary 
driver of outcomes, and that adding LVEF has limited value in prognostication, have been reported previously in patients with acute $\mathrm{HF}^{37}$ and in a recent community study, ${ }^{38}$ but these facts may also be true in chronic HFpEF. Natriuretic peptides could be used to predict outcomes in HFpEF patients by using adequate risk models. In our study, although we found no significant differences between the elderly and the nonelderly HFpEF patients regarding the natriuretic peptides (neither at baseline, nor at the 1-year follow-up), we observed that the predictive values for adverse outcomes were different for the two age groups. Readmissions due to $\mathrm{HF}$ aggravation were predicted by BNP levels $>450 \mathrm{pg} / \mathrm{mL}$ in the elderly $(\mathrm{OR}=1.004)$ and by BNP levels $>390 \mathrm{pg} / \mathrm{mL}$ in the nonelderly $(\mathrm{OR}=1.002)$.

Inflammatory biomarkers, including IL-6, TNF- $\alpha$, and hs-CRP, are associated with comorbidities, as well as with cardiac structural and functional changes in HF. They are elevated in HF patients and are associated with outcomes regardless of etiology. ${ }^{39}$ The elderly HFpEF patients included in our study presented with significantly higher levels of hs-CRP $(P=0.0001)$ and TNF- $\alpha(P=0.005)$ when compared to the nonelderly group. This fact could be explained by the higher prevalence of comorbidities in the elderly group. During the multivariate logistic regression analysis, we found that increased values of the inflammatory marker TNF- $\alpha$ were found to be significantly associated with total readmissions $(P<0.001)$ and readmissions due to HF aggravation in the nonelderly $(P<0.001)$, as well as with noncardiovascular readmissions in the elderly $(P=0.003)$. Our results are concordant with those of Putko et al. ${ }^{40}$ The authors found that the plasma levels of TNF- $\alpha$ and its two receptors (TNFR1 and TNFR2) were significantly elevated in HFpEF patients and significantly associated with the presence of comorbidities. TNFR2 levels were also significantly associated with an increasing grade of diastolic dysfunction and with the severity of symptoms in HFpEF. These results suggest that TNF- $\alpha$-mediated inflammation is comorbidity-driven and is a part of the pathogenesis of HFpEF. Since previous studies have failed to show that TNF- $\alpha$ antagonism could improve outcomes in HFpEF, new hypotheses have been developed regarding the inhibition of TNFR1 and the stimulation of TNFR2 as possible therapeutic options. ${ }^{41}$ Increased levels of the inflammatory marker IL-6 predicted, in the nonelderly HF group, the risk for total readmissions $(\mathrm{OR}=2.06 ; P=0.003)$, readmissions due to other cardiovascular causes $(\mathrm{OR}=0.53$; $P=0.045)$, and readmissions due to noncardiovascular causes $(\mathrm{OR}=0.27 ; P=0.039)$. In our study, hs-CRP was not retained as a predictive parameter for rehospitalization.
Regarding readmissions, the total rehospitalization rates during the 1-year follow-up period did not differ significantly between the two groups ( $73 \%$ vs $52 \%$, respectively; $P=0.101)$. The hospitalization rate due to aggravated HF was significantly higher in the elderly group ( $41 \%$ vs $6.8 \%$, respectively; $P=0.002$ ). In this group, total readmissions had the following independent predictors: BNP levels $>450 \mathrm{pg} / \mathrm{mL}(\mathrm{OR}=0.89 ; P<0.0001)$; a Fahrenberg risk score for depression of $>7.4(\mathrm{OR}=0.71$; $P=0.002)$; distances walked on the $6 \mathrm{MWT} \leq 248 \mathrm{~m}$ $(\mathrm{OR}=0.712 ; P=0.009) ;$ and TNF- $\alpha$ levels $\geq 10.1 \mathrm{pg} / \mathrm{mL}$ $(\mathrm{OR}=0.64 ; P=0.027)$. Independent predictors for readmissions due to cardiovascular causes in the elderly were NTproBNP levels $>458 \mathrm{pg} / \mathrm{mL}(\mathrm{OR}=1.002 ; P=0.001)$ and a Fahrenberg risk score for depression $>7.4(\mathrm{OR}=1.44$; $P=0.006)$. HF aggravation in the elderly was predicted by BNP levels $>450 \mathrm{pg} / \mathrm{mL}(\mathrm{OR}=1.004 ; P=0.007)$ and NT-proBNP levels $>477 \mathrm{pg} / \mathrm{mL}(\mathrm{OR}=1.002 ; P=0.013)$, while other cardiovascular readmissions were predicted by an MLHFQ score $>46(\mathrm{OR}=2.70 ; P=0.041)$ and IL-6 levels $>2.1 \mathrm{pg} / \mathrm{mL}$ $(\mathrm{OR}=0.53 ; P=0.047)$. The risk for noncardiovascular readmissions among the elderly was predicted by TNF- $\alpha$ levels $>10.1 \mathrm{pg} / \mathrm{mL}(\mathrm{OR}=1.30 ; P=0.003)$.

\section{Study limitations}

This study has several limitations, the most important of which is the small number of patients included at the single center. The advantage of this exclusively hospital-based design is that it allowed for the prospective recruitment of all eligible patients who had a first hospitalization for HF with preserved LVEF, as well as for their close follow-up for 1 year.

\section{Further developments}

Further studies with a focus on elderly people with HF are needed in order to outline the factors associated with readmission. A multidisciplinary approach with this high-risk HF group is necessary in order to improve life quality and reduce the burden of hospital readmissions. A strategy to improve the ambulatory care of HF patients could reduce both morbidity and mortality in this high-risk age group.

\section{Conclusion}

We conclude that in HFpEF patients aged $\geq 65$ years, the main cause of rehospitalization during the 1-year follow-up was HF aggravation. The risk for HF aggravation was predicted by increased levels of BNPs at baseline HF hospitalization, while noncardiovascular readmissions were 
significantly associated with increased levels of the inflammatory biomarker TNF- $\alpha$. Increased TNF- $\alpha$ levels predicted both cardiovascular and noncardiovascular readmissions, while increased levels of hs-CRP did not predict any of these outcomes in our study.

\section{Acknowledgments}

We would like to thank the attending physicians for filling their reports for all admitted patients; Mihaela Mot for imputing the patients' data into electronic databases; Ioana Citu for her support and contributions in the literature search strategy; Adina Bucur, Department of Public Health, for her helpful comments; and Emma Deak for her support in managing the project. English language editing of this manuscript was provided by Journal Prep.

\section{Author contributions}

$\mathrm{AMM}, \mathrm{DAB}$, and MCT participated in the conception and design of the study, statistical analysis and interpretation of the data, drafting of the article, and reviewing of the manuscript for intellectual content. TD and OA participated in interpretation of data, and in the design and drafting of the manuscript. IM performed the acquisition and interpretation of the data. All authors contributed toward data analysis, drafting and critically revising the paper and agree to be accountable for all aspects of the work.

\section{Disclosure}

The authors report no conflicts of interest in this work.

\section{References}

1. Lloyd-Jones D, Adams RJ, Brown TM, et al. Heart disease and stroke statistics - 2010 update: a report from the American Heart Association Statistics Committee and Stroke Statistics Subcommittee. Circulation. 2010;121:e1-e170.

2. Formiga F, Chivite D, Sole A. Functional outcomes of elderly patients after the first hospital admission for decompensated heart failure. A prospective study. Arch Gerontol Geriatr. 2006;43:175-185.

3. Borlaug BA, Redfield MM. Diastolic and systolic heart failure are distinct phenotypes within the heart failure spectrum. Circulation. 2011;123: 2006-2013.

4. Paulus WJ, Tschöpe C. A novel paradigm for heart failure with preserved ejection fraction: comorbidities drive myocardial dysfunction and remodeling through coronary microvascular endothelial inflammation. J Am Coll Cardiol. 2013;62:263-271.

5. Jugdutt BI. Aging and remodeling during healing of the wounded heart: current therapies and novel drug targets. Curr Drug Targets. 2008;9:325-344.

6. Paulus WJ, Tschope C, Sanderson J, et al. How to diagnose diastolic heart failure: a consensus statement on the diagnosis of heart failure with normal left ventricular ejection fraction by the Heart failure and Echocardiography Associations of the European Society of Cardiology. Eur Heart J. 2007;28:2539-2550.

7. National Kidney Foundation. K/DOQI clinical practice guidelines for chronic kidney disease: evaluation, classification, and stratification. Am J Kidney Dis. 2002;392(suppl 1):1-266.
8. McMurray J, Adamopoulos S, Anker SD, et al. ESC Guidelines for the diagnosis and treatment of acute and chronic heart failure 2012. Eur Heart J. 2012;33:1787-1847.

9. Montalescot G, Sechtern U, Achenbach S, et al. 2013 ESC guidelines on the management of stable coronary artery disease. Eur Heart J. 2013;34: 2949-3003.

10. Mancia G, Fagard R, Narkiewicz K, et al. 2013 ESH/ESC Guidelines for the management of arterial hypertension. Eur Heart J. 2013;34: 2159-2219.

11. Vahnian A, Alfieri O, Andreotti F, et al. Guidelines on the management of valvular heart disease (version 2012). Eur Heart J. 2012;33: 2451-2496.

12. Tendera M, Aboyans V, Bartelink AM, et al. ESC Guidelines on the diagnosis and treatment of peripheral artery diseases. Eur Heart $J$. 2011;32:2851-2906.

13. Alberti KG, Zimmet P, Shaw J. Metabolic syndrome-a new worldwide definition. A consensus statement from the international diabetes federation. Diabet Med. 2006;23:469-480.

14. Definition and Diagnosis of Diabetes Mellitus and Intermediate Hyperglycemia. Available from: https://www.idf.org/webdata/docs/ WHO_IDF_definition_diagnosis_of_diabetes.pdf. Accessed Dec 10, 2012.

15. Blanc B, Finch CA, Hallberg L, et al. Nutritional anaemias. Report of a WHO Scientific Group. WHO Tech Rep Ser. 1968;405: $1-40$.

16. Decramer M, Vestbo J, Bourbeau J, et al. Global Strategy for Diagnosis, Management, and Prevention of Chronic Obstructive Lung Disease (GOLD); 2013. Available from: www.goldcopd.org. Accessed Feb 20, 2013.

17. Lang RM, Bierig M, Devereux RB, et al; Chamber Quantification Writing Group; American Society of Echocardiography's Guidelines and Standards Committee; European Association of Echocardiography, Recommendation for chamber quantification: a report from the American Society of Echocardiography's Guidelines and Standards Committee and the Chamber Quantification Writing Group, Developed in Conjunction with the European Association of Echocardiography, a Branch of the European Society of Cardiology. J Am Soc Echocardiogr. 2005;18:1440-1463.

18. Rao A, Asadi-Lari M, Walsh J, Wilcox R, Gray D. Quality of life in patients with signs and symptoms of heart failure - does systolic function matter? J Card Fail. 2006;12:677-687.

19. Fahrenberg J, Myrtek M, Schumacher J, Brähler E. Fragebogen zur Lebenszufriedenheit (FLZ) [Questionnaire regarding life quality (Pocket Guide)]. Handanweisung. Göttingen: Hogrefe; 2000.

20. Demers C, McKelvie RS, Negassa A, Yusut S. Reliability, validity, and responsiveness of the six-minute walk test in patients with heart failure. Am Heart J. 2001;142:698-703.

21. Schwartz JB, Zipes DP. Cardiovascular disease in the elderly. In: Libby P, Bonow RO, Mann DL, Zipes DP, editors. Braunwald's Heart Disease: A Textbook of Cardiovascular Medicine. Vol. 2. Pennsylvania, PA: Saunders Elsevier; 2012:1727-1756.

22. Ahmed A, Rich MW, Fleg JL, et al. Effects of digoxin on morbidity and mortality in diastolic heart failure: the ancillary digitalis investigation group trial. Circulation. 2006;114:397-403.

23. Flather MD, Shibata MC, Coats AJ, et al; SENIORS Investigators. Randomized trial to determine the effect of nebivolol on mortality and cardiovascular hospital admission in elderly patients with heart failure (SENIORS). Eur Heart J. 2005;26:215-225.

24. Fonarow GC, Stough WG, Abraham WT, et al; OPTIMIZE-HF Investigators and Hospitals. Characteristics, treatments, and outcomes of patients with preserved systolic function hospitalized for heart failure: a report from the OPTIMIZE-HF Registry. $J$ Am Coll Cardiol. 2007;50:768-777.

25. World Health Organization. Definition of an older or elderly person. Geneva, Switzerland: WHO; 2010. Available from: http://www. who.int/healthinfo/survey/ageingdefnolder/en/index.html. Accessed November 22, 2010. 
26. Felker GM, Hasselblad V, Hernandez AF, O'Connor CM. Biomarkerguided therapy in chronic heart failure: a meta-analysis or randomized controlled trials associated with a significant reduction in all-cause mortality compared to usual care in patients with chronic heart failure. Am Heart J. 2009; 158:422-430.

27. Karogeropoulos A, Georgeopoulou V, Psaty BM. Inflammatory markers and incident heart failure risk in older adults: the health, aging, and body composition study. J Am Coll Cardiol. 2010;55(19):2129-2137.

28. Yusuf S, Pfeffer MA, Swedberg K, et al; CHARM Investigators and Committees. Effects of candesartan in patients with chronic heart failure and preserved left-ventricular ejection fraction: the CHARM-preserved trial. Lancet. 2003;362(9386):777-781.

29. Win S, Anand I, McMurray J, et al. Morbidity and mortality in diabetics with heart failure and a preserved ejection fraction. Result from the I-preserve trial. J Am Coll Cardiol. 2013;61(10_S):1498-1506.

30. Lenzen MJ, Scholte op Reimer WJ, Boersma E, et al. Differences between patients with a preserved and a depressed left ventricular function: a report from the EuroHeart Failure Survey. Eur Heart J. 2004; 25(14):1214-1220.

31. Hamdani N, Paulus WJ, van Heerebeek L, et al. Distinct myocardial effects of beta-blocker therapy in heart failure with normal and reduced left ventricular ejection fraction. Eur Heart J. 2009;30: 1863-1872.

32. Reil JC, Hohl M, Reil GH, et al. Heart rate reduction by If-inhibition improves vascular stiffness and left ventricular systolic and diastolic function in a mouse model of heart failure with preserved ejection fraction. Eur Heart J. 2013;34(36):2839-2949.

33. Kosmala W, Wang M, Wang T, et al. Effect of If-channel inhibition on hemodynamic status and exercise tolerance in heart failure with preserved ejection fraction: a randomized trial. JACC. 2013;62(15): $1330-1338$.
34. Dickstein K, Cohen-Solal A, Filippatos G, et al. ESC guidelines for the diagnosis and treatment of acute and chronic heart failure 2008: the Task Force for the diagnosis and treatment of acute and chronic heart failure 2008 of the European Society of Cardiology. Developed in collaboration with the Heart Failure Association of the ESC (HFA) and endorsed by the European Society of Intensive Care Medicine (ESICM). Eur J Heart Fail. 2008;10:933-989.

35. Paulus WJ, van Ballegoij JJ. Treatment of heart failure with normal ejection fraction: an inconvenient truth. J Am Coll Cardiol. 2010;55: 526-537.

36. Van Velhuisen DJ, Linssen GC, Jaarma T, et al. B-type natriuretic peptide and prognosis in heart failure patients with preserved and reduced ejection fraction. J Am Coll Cardiol. 2013;61(14):1498-1506.

37. Cleland JG, Tendera M, Adamus J, et al; PEP-CHF Investigators. The perindopril in elderly people with chronic heart failure (PEP-CHF) study. Eur Heart J. 2006;27:2338-2345.

38. Pitt B, Pfeffer MA, Assmann SF, et al; TOPCAT Investigators. Spironolactone for heart failure with preserved ejection fraction (TOPCAT). N Engl J Med. 2014;370:1383-1392.

39. Ghio S, Magrini G, Serio A, et al; SENIORS Investigators. Effects of nebivolol in elderly heart failure patients with or without systolic left ventricular dysfunction: results of the SENIORS echocardiographic substudy. Eur Heart J. 2006;27:562-568.

40. Putko BN, Wang Z, Lo J, et al, on behalf of the Alberta HEART Investigators. Circulating Levels of Tumor Necrosis Factor-Alpha Receptor 2 Are Increased in Heart Failure with Preserved Ejection Fraction Relative to Heart Failure with Reduced Ejection Fraction: Evidence for a Divergence in Pathophysiology. PLoS One. 2014;9(6):e99495.

41. Tehrani F, Phan A, Chien CV, Morrissey RP, Rafique AM, Schwarz ER. Value of medical therapy in patients $>80$ years of age with heart failure and preserved ejection fraction. Am J Cardiol. 2009;103:829-833.
Clinical Interventions in Aging

\section{Publish your work in this journal}

Clinical Interventions in Aging is an international, peer-reviewed journal focusing on evidence-based reports on the value or lack thereof of treatments intended to prevent or delay the onset of maladaptive correlates of aging in human beings. This journal is indexed on PubMed Central, MedLine,

\section{Dovepress}

CAS, Scopus and the Elsevier Bibliographic databases. The manuscript management system is completely online and includes a very quick and fair peer-review system, which is all easy to use. Visit http://www.dovepress. com/testimonials.php to read real quotes from published authors. 